\title{
475876 - SPINE ULTRASOUND FOR FACILITATING LABOR EPIDURALS IN OBESE PARTURIENTS
}

\author{
Yung Lee, MD ${ }^{1}$, Mrinalini Balki, MBBS, MD $^{1}$, Stephen H Halpern, MD $^{2}$, Jose CA \\ Carvalho, $\mathrm{MD}, \mathbf{P h D}^{1}$ \\ 1. Department of Anaesthesia, Mount Sinai Hospital, University of Toronto, \\ Toronto, ON, Canada \\ 2. Department of Anesthesia, Sunnybrook Health Sciences Centre, University of \\ Toronto, Toronto, ON, Canada
}

Introduction: Prepuncture lumbar ultrasound scanning has proven to be a reliable tool to facilitate labor epidural placement in non-obese parturients (1). Whether this method has the same reliability and accuracy in obese parturients is still unknown. In this study, we assessed the accuracy and reliability of prepuncture lumbar ultrasound scanning as a tool for epidural placement in obese parturients.

Methods: After REB approval at Mount Sinai Hospital, we enrolled 46 obese parturients, with a pre-pregnant body mass index $(\mathrm{BMI})>30 \mathrm{~kg} / \mathrm{m} 2$ who were requesting labor epidurals. Prepuncture lumbar ultrasound imaging (2-5 MHz curved array probe) was done with the patient in the sitting position, using the transverse approach, by one of the investigators. The anatomical landmarks, namely the midline and the intervertebral space, were identified, and the distance from the skin to the epidural space (ultrasound depth/UD) was estimated with a built-in caliper. An optimal needle insertion point was marked based on the obtained anatomical landmarks. Subsequently, an investigator blinded to the UD placed the epidural at the predetermined insertion point. During the epidural placement, the number of needle redirections and reinsertions made before successful epidural placement was recorded, and the actual distance from the skin to the epidural space (needle depth/ND) was marked on the Tuohy needle with a sterile marker. The correlation between the UD and ND was then studied using the Pearson's correlation coefficient and the Bland-Altman analysis.

Results: The mean \pm SD of maternal age was $31.7 \pm 7.3$ yr, pre-pregnancy BMI was 37.9 $\pm 9.3 \mathrm{~kg} / \mathrm{m} 2$ and BMI at delivery was $43.1 \pm 9.7 \mathrm{~kg} / \mathrm{m} 2$. The mean \pm SD of UD and ND were $6.3 \pm 0.8 \mathrm{~cm}$ and $6.6 \pm 0.98 \mathrm{~cm}$, respectively. The Pearson's correlation coefficient between UD and ND was 0.845 (95\% CI 0.735 - 0.911) [Figure 1]. The Bland-Altman analysis is shown in Figure 2, and shows a progressive underestimate of the distance measured by ultrasound with increasing needle depth. The epidural placement using the pre-determined insertion point was done without reinsertions in $76.1 \%$ of the cases, and there was no need to redirect the needle in $67.4 \%$ of the parturients.

Discussion: We found a strong correlation between the ultrasound-estimated distance and the actual measured needle distance in obese parturients. However, the ultrasound consistently underestimates the actual distance in a progressive fashion. This difference is likely due to the subcutaneous tissue compression during ultrasound scanning. We also found a good level of success using the ultrasound-determined insertion point for epidural needle placement. Our results suggest that prepuncture lumbar ultrasound can be a useful guide to facilitate labor epidural placement in obese parturients. 
References: 1. Arzola C, Davies S, Rofaeel A, Carvalho JCA. Ultrasound using the transverse approach to the lumbar spine provides reliable landmarks for labor epidurals. Anesth Analg 2007; 104:1188-92 